\title{
Spontaneous spinal epidural hematoma
}

\author{
Julia Röper-Kelmayr ${ }^{1}$, Eva Voglmayr ${ }^{1}$, Andreas Gruber ${ }^{2}$ and Franz A Fellner ${ }^{1,3}$ \\ ${ }^{1}$ Central Radiology Institute, Kepler University Hospital, Medical Faculty of the Johannes Kepler University, Linz, Austria \\ ${ }^{2}$ Department of Neurosurgery, Kepler University Hospital, Medical Faculty of the Johannes Kepler University, Linz, Austria \\ ${ }^{3}$ Medical Faculty of the Friedrich-Alexander-University of Erlangen-Nürnberg, Erlangen, Germany
}

\section{Case report}

A 31-year-old male came into our clinic's emergency room complaining of a somatosensory disorder that had worsened considerably in the previous few hours as well as motor dysfunction in the right upper and lower extremities. The patient also reported sudden, initially acute pain in the back of his neck on the right side. This condition had definitely not been the result of trauma.

Due to the rapidly progressing symptoms, the patient was sent, on an urgent basis, for an MR imaging scan of the entire neurological axis.

This revealed a circumscribed lesion in the spinal canal dorsolateral right epidural at $\mathrm{C} 3$ to $\mathrm{C} 5$, which, in relation to the spinal cord, was situated isointense in T1-weighted scans and hyperintense in T2weighted scans, and displayed absolutely no pathological bone marrow impregnation (Figure 1). The images produced by the MR scan gave rise to suspicion of a spontaneous spinal epidural hematoma. The lesion

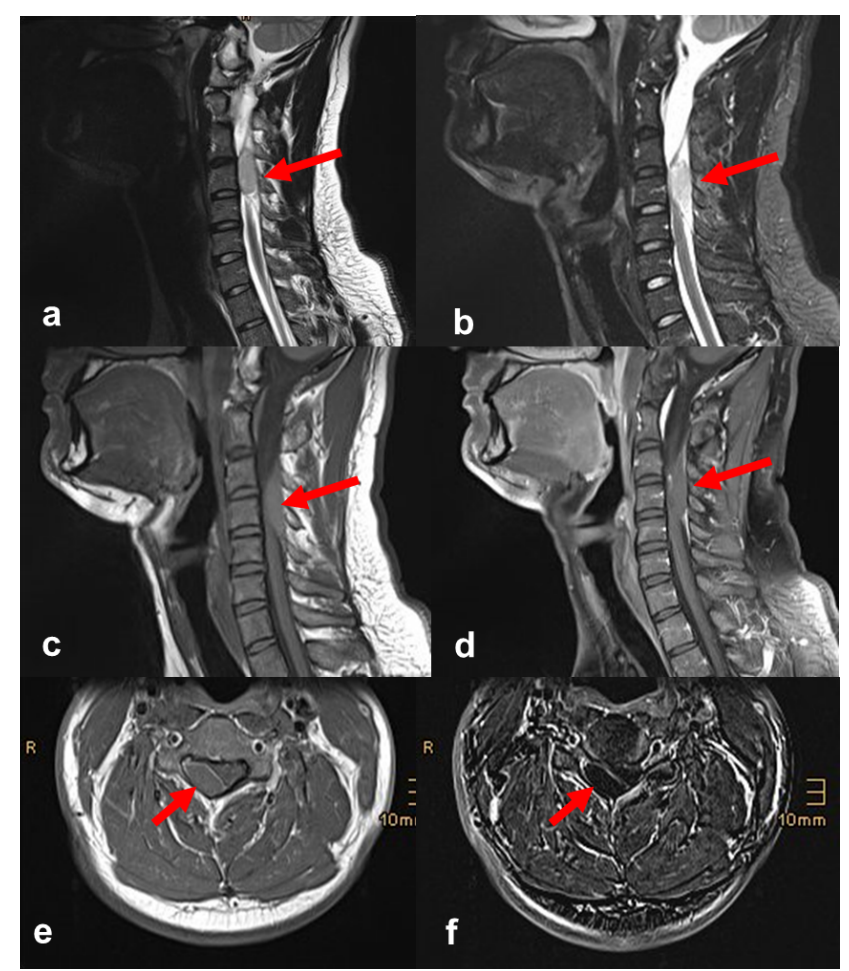

Figure 1. Spontaneous spinal epidural hematoma exerting pressure on the spinal cord. MR imaging: sagittal T2-weighted turbo spin-echo (a), T2-weighted STIR (b), T1-weighted turbo spin-echo before (c) and after intravenous administration of contrast agent (d), transverse T1-weighted turbo spin-echo after contrast agent administration (e), as well as T1-weighted subtracted image (f). was noticeably exerting pressure on the spinal cord. At the time of the examination, the MR scan provided no indication of myelopathy.

Thereafter, neurosurgical decompression was performed via hemilaminectomy and hematoma evacuation. Immediately after these procedures were performed, the neurological symptoms subsided substantially.

\section{Discussion}

Spontaneous spinal epidural bleeding - defined as epidural bleeding in the absence of a traumatic or iatrogenic cause - is a rare occurrence with an incidence of $0.1 / 100,000$ per year [1]. Men are more frequently affected than women [2].

Among the risk factors cited for the occurrence of a spontaneous epidural hematoma are the ingestion of anticoagulants, coagulopathies, vascular malformations and trivial traumas $[1,2]$. However, in most cases - including that of our patient - no underlying cause is found.

The imaging method of choice is magnetic resonance imaging, which makes it possible to draw conclusions with respect to not only the location and extent of the bleeding but also how old the hematoma is. Thus, acute bleeding reveals in T1-weighted scans isointense and in T2-weighted scans hyperintense signal with hypointense foci; subacute bleeding in T1- and T2-weighted scans exhibits a hyperintense signal $[2,3]$.

In the differential diagnosis, an intraspinal tumor or an abscess formation must be considered, whereby the contrast medium facilitated the diagnosis in this case. Fat saturated sequences help to differentiate between a hematoma and epidural fat [1].

\section{Disclosure}

No conflicts of interest. No grants or financial support.

\section{References}

1. Figueroa J, DeVine JG (2017) Spontaneous spinal epidural hematoma: Literature review. J Spine Surg 3: 58-63. [Crossref]

Correspondence to: Julia Röper-Kelmayr, Central Radiology Institute, Kepler University Hospital, Medical Faculty of the Johannes Kepler University, Linz, Austria, E-mail: julia.roeper-kelmayr@kepleruniklinikum.at

Key words: spontaneous spinal hematoma, cervical spine, magnetic resonance imaging

Received: January 09, 2018; Accepted: January 25, 2018; Published: January 27, 2018 
2. Firsching R, Zanella F, Lanfermann H (1993) The dynamics of magnetic resonance findings in patients with a spinal haematoma. Paraplegia 31: 180-185. [Crossref]
3. Fukui MB, Swarnkar AS, Williams RL (1999) Acute spontaneous spinal epidural hematomas. AJNR Am J Neuroradiol 20: 1365-1372. [Crossref]

Copyright: $(02018$ Röper-Kelmayr J. This is an open-access article distributed under the terms of the Creative Commons Attribution License, which permits unrestricted use, distribution, and reproduction in any medium, provided the original author and source are credited. 